\title{
Vortex Motion Quantifies Strong Dissipation in a Holographic Superfluid
}

\author{
Paul Wittmer $\odot,{ }^{1,2, *}$ Christian-Marcel Schmied, ${ }^{2,3, *}$ Thomas Gasenzer $\odot,{ }^{1,2,3}$ and Carlo Ewerz $\oplus^{1,2}$ \\ ${ }^{1}$ Institut für Theoretische Physik, Universität Heidelberg, Philosophenweg 16, 69120 Heidelberg, Germany \\ ${ }^{2}$ ExtreMe Matter Institute EMMI, GSI Helmholtzzentrum für Schwerionenforschung, Planckstraße 1, 64291 Darmstadt, Germany \\ ${ }^{3}$ Kirchhoff-Institut für Physik, Universität Heidelberg, Im Neuenheimer Feld 227, 69120 Heidelberg, Germany
}

(Received 21 December 2020; accepted 23 June 2021; published 31 August 2021)

\begin{abstract}
Holographic duality provides a description of strongly coupled quantum systems in terms of weakly coupled gravitational theories in a higher-dimensional space. It is a challenge, however, to quantitatively determine the physical parameters of the quantum systems corresponding to generic holographic theories. Here, we address this problem for the two-dimensional holographic superfluid, known to exhibit strong dissipation. We numerically simulate the motion of a vortex dipole and perform a high-precision matching of the corresponding dynamics resulting from the dissipative Gross-Pitaevskii equation. Excellent agreement is found for the vortex core shape and the spatiotemporal trajectories. A further comparison to the Hall-Vinen-Iordanskii equations for point vortices interacting with the superfluid allows us to determine the friction parameters of the holographic superfluid. Our results suggest that holographic vortex dynamics can be applied to experimentally accessible superfluids like strongly coupled ultracold Bose gases or thin helium films with temperatures in the Kelvin range. This would make holographic far-fromequilibrium dynamics and turbulence amenable to experimental tests.
\end{abstract}

DOI: 10.1103/PhysRevLett.127.101601

Introduction.-The time evolution of quantum manybody systems out of equilibrium has attracted considerable attention in recent years [1-8]. Strong correlations prevailing in these systems generically necessitate nonperturbative methods to quantitatively describe the dynamics. In particular the interplay between linear and strongly nonlinear excitations such as topological defects poses a challenge for theory which is even amplified for strongly coupled and dissipative systems.

Holography [9-11], also known as gauge-gravity duality, allows one to address such problems in an intrinsically nonperturbative framework. It posits the equivalence of certain quantum field theories to gravitational theories with an additional dimension of space. In bottom-up constructions of this duality, a strongly coupled field theory is mapped to weakly coupled, and hence classical, Einstein gravity on an anti-de Sitter (AdS) space. Finite temperature of the field theory corresponds to a black hole on the gravity side. By now, a wide range of holographic dualities has been established, with applications ranging from nuclear to condensed matter physics (see, e.g., Refs. [12-16] for reviews). While the systems described by generic bottomup holographic models are qualitatively known to be

Published by the American Physical Society under the terms of the Creative Commons Attribution 4.0 International license. Further distribution of this work must maintain attribution to the author(s) and the published article's title, journal citation, and DOI. Funded by SCOAP. strongly coupled, their field-theory Lagrangians are not known and it has proven notoriously difficult to quantitatively determine their coupling and phenomenological parameters.

Here, we address this long-standing problem for the holographic model of a superfluid in two spatial dimensions [17-20]. This system has been studied extensively, with particular focus on linear [18,19,21,22] and nonlinear excitations such as vortices [23-29]. So far it was unknown how the holographic superfluid compares to experimentally realized superfluids like ultracold atomic gases [30] or liquid helium [31].

Specifically, we aim at a quantitative characterization of vortex dynamics in the holographic superfluid. For that we study the time evolution of a vortex-antivortex pair in holography and in the dissipative Gross-Pitaevskii equation (DGPE) [32]. By tuning the DGPE parameters, we match the holographic vortices' core shape as well as their trajectories in space and time [33]. We furthermore use known relations between the DGPE and the HallVinen-Iordanskii (HVI) equations [34-36] describing the mechanical motion of point vortices subject to interactions with the superfluid. This allows extracting friction coefficients of vortices in the holographic superfluid which we compare to those of experimentally accessible superfluids. In the following we present our main results. For technical details see the Supplemental Material [37].

Superfluidity is a low-temperature phenomenon associated with Bose-Einstein condensation. The condensate is described by a nonzero expectation value of a bosonic field 
operator, $\langle\Psi(\mathbf{r}, t)\rangle \neq 0$. The classical field $\psi(\mathbf{r}, t)=$ $\langle\Psi(\mathbf{r}, t)\rangle=\sqrt{\rho(\mathbf{r}, t)} \exp \{\mathrm{i} \theta(\mathbf{r}, t)\}$ encodes the density $\rho=|\psi|^{2}$ of the condensed particles and their velocity field $\mathbf{v}(\mathbf{r}, t)=\nabla \theta(\mathbf{r}, t) . \psi$ acts as an order parameter for the superfluid phase. The Tisza-Landau two-fluid model $[93,94]$ offers a successful description of superfluidity by invoking a second, "normal" (or thermal) component of the total liquid and accounting for its interaction with the superfluid condensate. We denote the thermal equilibrium condensate density as $\rho_{0}$ and use it as a background for imprinting vortices. The high fluid velocity close to a vortex core leads to friction between the superfluid and the normal component, characterized by friction coefficients that we study here.

In holography, the simplest bottom-up gravitational description of a $(2+1)$-dimensional superfluid is given by an Abelian Higgs model,

$$
\mathcal{L}_{\text {gauge-matter }}=-\frac{1}{4} F_{\mu \nu} F^{\mu \nu}-\left|D_{\mu} \Phi\right|^{2}-m^{2}|\Phi|^{2},
$$

on a $(3+1)$-dimensional (asymptotically) AdS spacetime with a black hole [17-19]. In the probe limit, this Schwarzschild-AdS background is static and has curvature radius $L_{\mathrm{AdS}}$ and horizon position $z_{h}$,

$d s^{2}=\frac{L_{\mathrm{AdS}}^{2}}{z^{2}}\left[-\left(1-\frac{z^{3}}{z_{h}^{3}}\right) d t^{2}+d x^{2}+d y^{2}-2 d t d z\right]$.

$t, x, y$ are the superfluid's coordinates, $z$ is the additional holographic coordinate, and we write $\mathbf{r}=(x, y) . \mu, \nu=t$, $x, y, z$ specify the vector field's components. The matter part (1) contains a scalar field $\Phi$ with mass $m$, the field strength tensor $F_{\mu \nu}=\nabla_{\mu} A_{\nu}-\nabla_{\nu} A_{\mu}$ of the gauge field $A_{\mu}$, and the gauge-covariant derivative $D_{\mu}=\nabla_{\mu}-i A_{\mu}$. The probe limit assumed here is valid at sufficiently high temperatures where the energy of the gauge-matter sector is small enough to neglect its backreaction on the AdS spacetime. To study very low temperatures, the geometry (2) would need to be modified [95].

Spontaneous symmetry breaking occurs in the holographic superfluid due to condensation of the scalar field near the black hole and formation of a charge cloud in the bulk of the AdS spacetime, screening the boundary from the black hole [17]. The superfluid can be thought of as a projection of the bulk dynamics onto the boundary of the spacetime at $z=0$, with the quantum expectation value $\psi=\langle\Psi\rangle$ obtained from a near-boundary expansion of the dual field $\Phi$ under appropriate boundary conditions, $\Phi(t, \mathbf{r}, z)=\psi(\mathbf{r}, t) z^{2}+\mathcal{O}\left(z^{3}\right)$. Figure 1 illustrates the bulk picture. In the duality, the black hole corresponds to a static heat bath with temperature $\tilde{T}=3 /\left(4 \pi z_{h}\right)$ and may be considered as the normal component of the system, whereas the gauge-matter sector corresponds to the superfluid component. The chemical potential of the superfluid

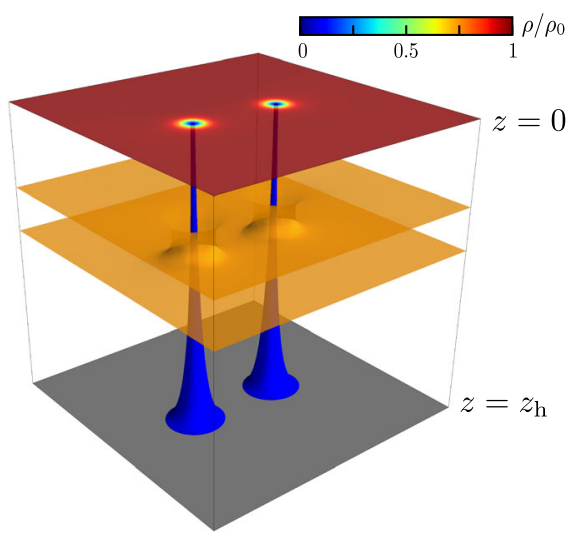

FIG. 1. Visualization of the bulk configuration of the holographic superfluid in the presence of a vortex-antivortex pair. The blue tubes are isosurfaces of the scalar field $|\Phi|^{2} / z^{4}$, which reduces to the superfluid density $\rho$ at the boundary $(z=0)$. The normalized superfluid density $\rho / \rho_{0}$ is encoded according to the color scale. Isosurfaces of the scalar charge cloud in the bulk are illustrated by the two orange sheets which the vortex tubes pierce through. The gray area at $z=z_{h}$ represents the black hole horizon.

is fixed by the boundary condition of the temporal gauge field component, $\tilde{\mu}=A_{t}(z=0)$ [96]. Notably, $\tilde{T}$ and $\tilde{\mu}$ are not independent here. In our numerical simulations we choose $z_{h} \equiv 1$ such that $\tilde{\mu}$ is the only free parameter, which sets the system into the superfluid phase above a critical value $\tilde{\mu}_{\mathrm{c}} \simeq 4.064$ [19]. It further fixes the ratio $\tilde{T} / \tilde{T}_{c}$ via $\tilde{\mu} \tilde{T} / \tilde{T}_{c}=\tilde{\mu}_{c}$.

Dissipative Gross-Pitaevskii model.-A dilute cold Bose gas subject to damping due to interactions between the condensate and the non-condensate excitations can be described by the DGPE for the order-parameter field $\psi(\mathbf{r}, t)$. For a single-component system it reads

$\partial_{t} \psi(\mathbf{r}, t)=-(\mathrm{i}+\gamma)\left[-\frac{1}{2 M} \nabla^{2}+g|\psi(\mathbf{r}, t)|^{2}-\mu\right] \psi(\mathbf{r}, t)$,

where $M$ is the mass of the bosonic particles. The coupling parameter $g$ characterizes the interactions of the particles, and $\mu$ is a chemical potential representing a constant shift of the single-particle energy. The dimensionless phenomenological damping parameter $\gamma$ quantifies the dissipation. Values of $\gamma \gg 0.01$ are considered to describe strong dissipation. We take $\mu=g \rho_{0}$ such that the zero mode is not damped. The healing length $\xi$, the characteristic length scale set by the interactions, is then given by $\xi=(2 M \mu)^{-1 / 2}$. Because of the physical scale $\xi$ any nontrivial scale transformation would lead to a different physical system.

Quantized vortices represent topological structures in the complex field $\psi$. Around a vortex core, the phase of the 
order parameter winds by $2 \pi w_{i}$, with winding number $w_{i} \in \mathbb{Z} \backslash\{0\}$. Consequently, the density $\rho$ at the position of the core drops to zero, within a distance on the order of $\xi$. At large distances from the core, $\rho$ approaches the background density $\rho_{0}$.

Numerical solution and matching procedure.-Both the holographic and DGPE systems are numerically solved on grids with $512 \times 512$ grid points in the $(x, y)$ direction subject to periodic boundary conditions. For the holographic system, we additionally employ 32 collocation points along the $z$ direction. We consider several values of $\tilde{\mu}$ for which the probe limit is justified. For given $\tilde{\mu}$, we then adjust the numerical parameters in the DGPE simulations to match the vortices' sizes and space-time trajectories from holography.

To match the vortex sizes, we imprint a symmetric and periodic, and hence static, $2 \times 2$ configuration of two vortices and two antivortices with winding numbers \pm 1 into a homogeneous condensate background $\rho_{0}$. (This configuration is used only for this purpose.) We tune the DGPE healing length $\xi$ to adjust the size of the vortices to the holographic ones.

Using $\xi$ determined from the vortex sizes, we then study the propagation of one vortex-antivortex pair which without friction would move perpendicular to the dipole vector d connecting the vortex positions, a motion known as Helmholtz pair propagation. In the presence of friction between the defects and the fluid, the vortices are slowed relative to the superfluid flow such that a Magnus force emerges, causing a velocity component parallel to $\mathbf{d}$. As a result, the vortices approach each other and annihilate into a rapidly decaying arc wave. The strong suppression of Helmholtz pair propagation seen in our simulation is a direct consequence of the system losing energy to the thermal bath via dissipation. We determine the trajectories on the grid with subplaquette resolution in a quasicontinuous manner by a combination of two tracking methods, cf. Ref. [29]: a two-dimensional Gaussian fit to the density depressions around the vortex cores and a Newton-Raphson algorithm for tracking the zeros in the superfluid density. The precision of this procedure allows us to match the DGPE vortex trajectories in space and time by adjusting the dissipation constant $\gamma$ and time rescaling parameter $\tau$. (The latter defines the DGPE grid unit of time relative to the spatial grid unit $s$ by $t \sim M s^{2} / \tau$.)

With the knowledge of the DGPE parameters, we can fit solutions of the HVI equations for the motion of point vortices to the dipole trajectories to obtain friction coefficients of the holographic superfluid. We do this in a regime where artifacts due to the preparation of the initial vortex configuration (in particular a slight initial outward bending of the trajectories [29]) have died out but the vortices are still far enough apart.

Results.-Matching the sizes of the vortices, we find also their shapes in remarkable agreement, see Fig. 2. We furthermore obtain that the directly fitted width $\tilde{\xi}$ of the

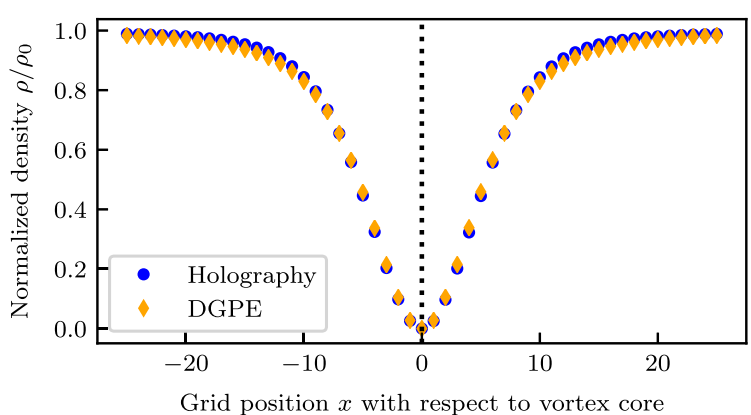

FIG. 2. Normalized density profile $\rho / \rho_{0}$ of a single vortex on a one-dimensional cut through its center, in holography $(\tilde{\mu}=6)$ and DGPE. The vortex shapes agree within $2 \%$ of the background density.

holographic vortex cores scales as $\tilde{\xi} \sim\left(\tilde{\mu}-\tilde{\mu}_{0}\right)^{-1 / 2}$, with the shift $\tilde{\mu}_{0} \simeq 4.06$ being close to the critical chemical potential $\tilde{\mu}_{c} \simeq 4.064$, as is typical for nonrelativistic superfluids.

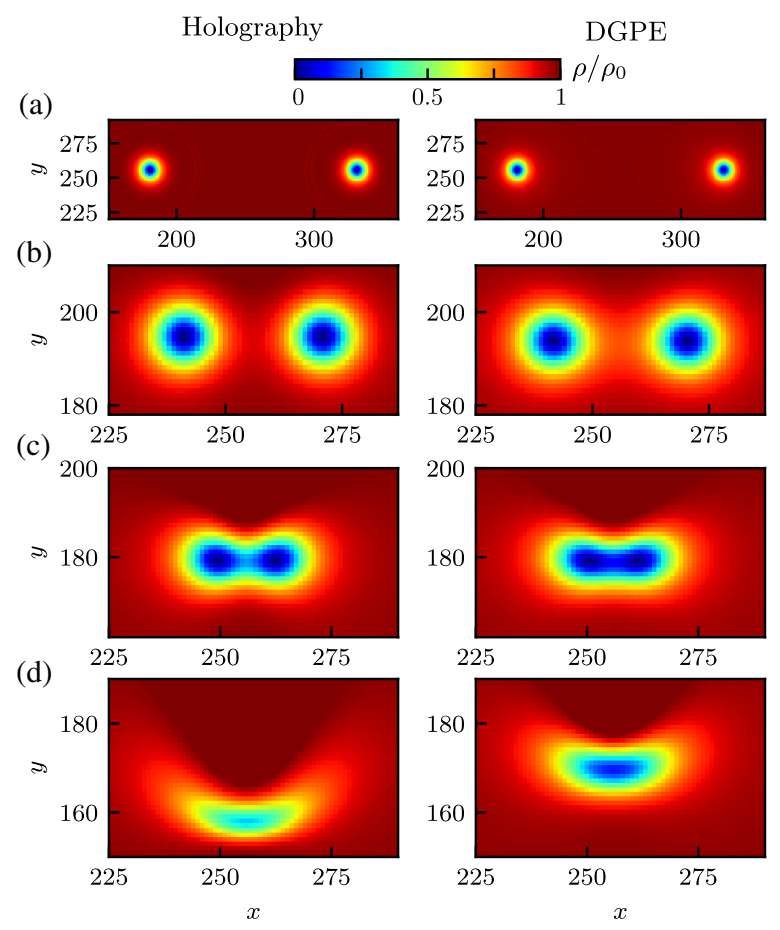

FIG. 3. Snapshots of the normalized superfluid density profile $\rho / \rho_{0}$ showing the vortex dipole in holography (left panels) and DGPE (right panels) at four different times. Note the different subregions of the $(x, y)$ grid. We find good agreement until shortly before the annihilation. (a) Initial configuration: The vortices have a circular shape and are well separated. (b) Intermediate stage: The vortices approach each other and start to be deformed to an elliptical shape. (c) Shortly before annihilation: The vortices are strongly deformed and their density suppressions overlap. At this stage the dynamics can no longer be matched and the trajectories shown in Fig. 4 end. (d) After annihilation: shock waves propagate through the fluid and quickly decay, with clear differences in size and shape between holography and DGPE. 


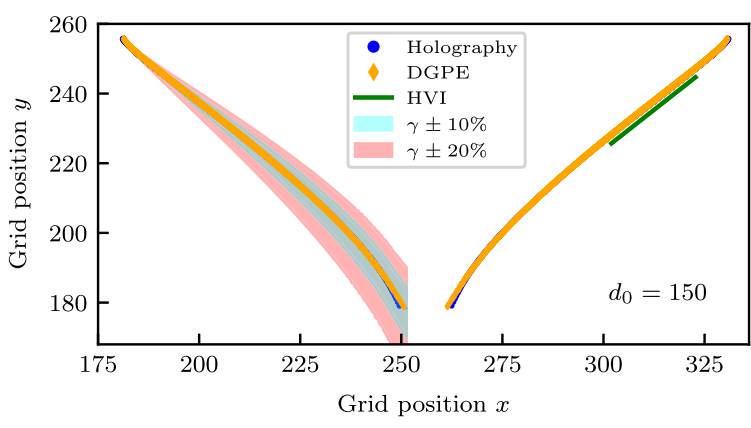

FIG. 4. Vortex trajectories in holography for $\tilde{\mu}=6$ (blue dots) and in DGPE (orange diamonds) for an initial vortex dipole separation of $d_{0}=150$ grid points. Except just before the annihilation of the dipole, we find remarkable agreement between the trajectories. The error bands correspond to variations of the damping parameter $\gamma$ by $10 \%$ (turquoise) and $20 \%$ (red). We compare our data to the motion calculated from the HVI equations in a suitable (linear) regime (green solid line, shifted downwards by -2.5 grid points).

For the example of $\tilde{\mu}=6$ and an initial separation of $d_{0}=150$ grid points, Fig. 3 displays four characteristic snapshots of the superfluid density distributions in the holographic and DGPE simulations of the dipole. Figure 4 demonstrates that the matched vortex trajectories agree very well until shortly before the annihilation. During the final stage of the annihilation process, the vortices deform and accelerate strongly. We show the trajectories up to a few timesteps before the annihilation, at which point the matching procedure ceases to work. The shock waves after the annihilation behave markedly different in the two theories.

Table I summarizes our results for the DGPE and HVI parameters matching the holographic dynamics for different $\tilde{\mu}$. We note that the values of $\gamma$ are relatively large for the approximation underlying the DGPE. The DGPE can nevertheless be used as an effective phenomenological description of vortex dynamics, for which the consistent matching provides evidence.

TABLE I. DGPE parameters (middle columns) for matching the holographic vortex shapes and trajectories for various choices (left columns) of $\tilde{\mu}$, or, equivalently, $\tilde{T} / \tilde{T}_{c}$. The right columns show the dimensionless friction coefficients, $C=1-\left(1+\gamma^{2}\right) /$ $\left[2 \tau\left(1+\gamma^{2}|\ln \xi|^{2}\right)\right]$ and $C^{\prime}=\gamma|\ln \xi|\left(1+\gamma^{2}\right) /\left[2 \tau\left(1+\gamma^{2}|\ln \xi|^{2}\right)\right]$ of the HVI equations, evaluated on a scaled grid with $\xi \rightarrow \xi / \hat{s}$. We estimate the errors of all extracted parameters to be on the order of $1 \%-2 \%$.

\begin{tabular}{llcccccc}
\hline \hline$\tilde{\mu}$ & $\tilde{T} / \tilde{T}_{c}$ & $\gamma$ & $\xi$ & $\tau$ & $\hat{s}$ & $C$ & $C^{\prime}$ \\
\hline 4.5 & 0.9 & 0.330 & 9.25 & 2.85 & 114 & 0.88 & 0.095 \\
6 & 0.68 & 0.313 & 3.50 & 5.76 & 116 & 0.95 & 0.047 \\
7.5 & 0.54 & 0.297 & 2.56 & 7.46 & 116 & 0.97 & 0.036 \\
9 & 0.45 & 0.281 & 1.93 & 9.25 & 119 & 0.98 & 0.029 \\
\hline \hline
\end{tabular}

Dissipation is a typical feature of fluids and can be well described in holographic hydrodynamics, see, e.g., Ref. [14], which also applies to superfluids. Interestingly, with vortices present in the holographic superfluid an additional contribution to dissipation can be observed which has a geometric interpretation. Vortices correspond to tubes with vanishing $\Phi$ along their axis which punch holes through the charge cloud (see Fig. 1) and allow for excitations of the boundary superfluid to fall into the black hole, thus dissipating energy to the black hole [26]. Because of the finite width of the holes, this favors the dissipation of short-wavelength modes of the superfluid, while the DGPE uniformly damps all modes except the zero mode. The holographic dissipation through vortex cores naturally stops with the annihilation of the vortices. The observed difference in the arc waves after the vortex annihilation demonstrates a difference in the relative strength of dissipation with and without vortices in holography and DGPE. This suggests that the inherently strongly coupled dissipation mechanism of the holographic framework is not fully captured by the DGPE.

Real-world superfluids.-Having quantified the dissipation and friction parameters of the two-dimensional holographic superfluid, we compare them to experimentally accessible superfluids.

Ultracold, dilute Bose condensates, well described within the Gross-Pitaevskii framework, are typically prepared with alkali atoms. In experiments with a quasi two-dimensional trapping geometry, disc-shaped clouds have been realized, with a thickness of $\sigma \sim 10^{2} \ldots 10^{3} \mathrm{~nm}$ $[2,3,97]$. In such systems, collisions are captured by the coupling $g=\sqrt{8 \pi} a /(M \sigma)$ [97-99], where $a$ is the threedimensional $s$-wave scattering length taking values of $a \simeq$ $3 \mathrm{~nm}$ and $5 \mathrm{~nm}$ for ${ }^{23} \mathrm{Na}$ and ${ }^{87} \mathrm{Rb}$, respectively. Typical experimental surface densities of $\rho \sim 10^{13} \mathrm{~m}^{-2}[2,3,97]$ lead to healing lengths on the order of $\xi=(4 \sqrt{2 \pi} a \rho / \sigma)^{-1 / 2} \sim$ $0.4 \ldots 2 \mu \mathrm{m}$. The quasi two-dimensional experimental settings satisfy the conditions for the applicability of the DGPE (3) as the dimensionless parameter $\zeta_{2 \mathrm{D}} \sim a /(\sqrt{2 \pi} \sigma)$ is small. If the temperature $T$ of the condensed Bose gas is large compared to the zero-point energy but well below the critical temperature of the Berezinskii-Kosterlitz-Thouless transition $[100,101]$, the damping $\gamma$ originates from the interactions between the condensed and thermal atoms. This gives rise to a self-energy shift which in leading approximation implies $\gamma \simeq 12 M a^{2} T / \pi$ [102]. For typical experimental temperatures of order $T \sim 100 \mathrm{nK}$, one infers $\gamma \sim 10^{-4} \ldots 10^{-3}$ which is 2 to 3 orders of magnitude smaller than the dissipation scale obtained above for the holographic model, see Table I. To induce such a strong dissipation in an alkali gas would require increasing the scattering length, e.g., close to a Feshbach resonance while decreasing the density $\rho$ to tune $\mu=\sqrt{8 \pi} a \rho /(M \sigma)$ and thus $\xi$ to the value matching the holographic model. In the experimental setups realized so far, this requires $a \sim \sigma$. Typically, such a system is difficult to be 
stabilized with bosons due to three-body-recombination loss prevailing at large scattering lengths [103], which, however, can be remedied by using ultracold paired fermions [104].

It is interesting to note that friction coefficients $C^{\prime}$ in the range quoted in our Table I have been reported in thermally excited atomic Bose condensates at $T \sim 300 \mathrm{nK}, C^{\prime} \sim$ $0.01 \ldots 0.03$ [105].

Finally, it is compelling to compare our results with measurements of vortex diffusivities in thin films of superfluid ${ }^{4} \mathrm{He}[1,7,106,107]$. Here, values of the HVI friction coefficient $C^{\prime}$ very similar to our results for the holographic superfluid have indeed been measured in helium films at temperatures of order $T \sim 1 \mathrm{~K}, C^{\prime} \sim 10^{-2} \ldots 1$ [106-109]. Note that in these experiments the temperature at which a certain $C^{\prime}$ is observed also depends on the thickness of the film.

Summary.-Holography provides a description of a strongly dissipative superfluid in terms of a higherdimensional gravitational theory. In this Letter, we have performed a high-precision study of the dynamics of a vortex dipole. We have matched the quantum vortex dynamics of the DGPE as well as the vortex mechanics of the HVI equations to the two-dimensional holographic superfluid, thereby quantifying its dissipation. The strongly dissipative character of the holographic superfluid is confirmed by the extracted values of the DGPE damping parameter $\gamma$ and of the HVI friction coefficient $C^{\prime}$. Our findings suggest that holographic vortex dynamics can be applied to and be tested in experimentally accessible superfluids. Candidates for the experimental realization are strongly coupled Bose gases and, in particular, thin helium films with temperatures in the Kelvin range. While we have derived these results from a simple vortex configuration, we expect holography to be applicable also to more complicated vortex ensembles, or turbulence in general, in strongly dissipative superfluids.

We thank C. Barenghi, R. Carretero-González, M. Karl, P. G. Kevrekidis, and A. Samberg for discussions and collaboration. This work was supported by EU Horizon2020 (ERC Adv. Grant EntangleGen, Project-ID 694561), by DFG (SFB 1225 ISOQUANT), and by Heidelberg University (CQD). P. W. was supported by the Studienstiftung des deutschen Volkes e. V.

*These authors contributed equally to this work.

[1] Y. P. Sachkou, C. G. Baker, G. I. Harris, O. R. Stockdale, S. Forstner, M. T. Reeves, X. He, D. L. McAuslan, A. S. Bradley, M. J. Davis et al., Science 366, 1480 (2019).

[2] S. P. Johnstone, A. J. Groszek, P. T. Starkey, C. J. Billington, T. P. Simula, and K. Helmerson, Science 364, 1267 (2019).

[3] G. Gauthier, M. T. Reeves, X. Yu, A. S. Bradley, M. A. Baker, T. A. Bell, H. Rubinsztein-Dunlop, M. J. Davis, and T. W. Neely, Science 364, 1264 (2019).
[4] M. Prüfer, P. Kunkel, H. Strobel, S. Lannig, D. Linnemann, C.-M. Schmied, J. Berges, T. Gasenzer, and M. K. Oberthaler, Nature (London) 563, 217 (2018).

[5] C. Eigen, J. A. P. Glidden, R. Lopes, E. A. Cornell, R. P. Smith, and Z. Hadzibabic, Nature (London) 563, 221 (2018).

[6] S. Erne, R. Bücker, T. Gasenzer, J. Berges, and J. Schmiedmayer, Nature (London) 563, 225 (2018).

[7] G. I. Harris, D. L. McAuslan, E. Sheridan, Y. Sachkou, C. Baker, and W. P. Bowen, Nat. Phys. 12, 788 (2016).

[8] L. Chomaz, L. Corman, T. Bienaimé, R. Desbuquois, C. Weitenberg, S. Nascimbène, J. Beugnon, and J. Dalibard, Nat. Commun. 6, 6162 (2015).

[9] J. Maldacena, Int. J. Theor. Phys. 38, 1113 (1999).

[10] S. Gubser, I. Klebanov, and A. Polyakov, Phys. Lett. B 428, 105 (1998).

[11] E. Witten, Adv. Theor. Math. Phys. 2, 253 (1998).

[12] A. Adams, L. D. Carr, T. Schäfer, P. Steinberg, and J. E. Thomas, New J. Phys. 14, 115009 (2012).

[13] J. Casalderrey-Solana, H. Liu, D. Mateos, K. Rajagopal, and U. A. Wiedemann, Gauge/String Duality, Hot QCD and Heavy Ion Collisions (Cambridge University Press, Cambridge, England, 2014).

[14] S. A. Hartnoll, Classical Quantum Gravity 26, 224002 (2009).

[15] J. McGreevy, Adv. High Energy Phys. 2010, 723105 (2010).

[16] J. Zaanen, Y.-W. Sun, Y. Liu, and K. Schalm, Holographic Duality in Condensed Matter Physics (Cambridge University Press, Cambridge, England, 2015).

[17] S. S. Gubser, Phys. Rev. D 78, 065034 (2008).

[18] S. A. Hartnoll, C. P. Herzog, and G. T. Horowitz, Phys. Rev. Lett. 101, 031601 (2008).

[19] C. P. Herzog, P. K. Kovtun, and D. T. Son, Phys. Rev. D 79, 066002 (2009).

[20] S. A. Hartnoll, A. Lucas, and S. Sachdev, arXiv:1612 .07324 .

[21] J. Sonner and B. Withers, Phys. Rev. D 82, 026001 (2010).

[22] D. Anninos, S. A. Hartnoll, and N. Iqbal, Phys. Rev. D 82, 066008 (2010).

[23] M. J. Bhaseen, J. P. Gauntlett, B. D. Simons, J. Sonner, and T. Wiseman, Phys. Rev. Lett. 110, 015301 (2013).

[24] V. Keränen, E. Keski-Vakkuri, S. Nowling, and K. P. Yogendran, Phys. Rev. D 81, 126012 (2010).

[25] O. J. C. Dias, G. T. Horowitz, N. Iqbal, and J. E. Santos, J. High Energy Phys. 04 (2014) 096.

[26] A. Adams, P. M. Chesler, and H. Liu, Science 341, 368 (2013).

[27] C. Ewerz, T. Gasenzer, M. Karl, and A. Samberg, J. High Energy Phys. 05 (2015) 070.

[28] S.-Q. Lan, G.-Q. Li, J.-X. Mo, and X.-B. Xu, J. High Energy Phys. 02 (2019) 122.

[29] C. Ewerz, A. Samberg, and P. Wittmer, arXiv:2012.08716.

[30] L. P. Pitaevskii and S. Stringari, Bose-Einstein Condensation (Clarendon Press, Oxford, 2003).

[31] R. J. Donnelly, Quantized Vortices in Helium II, Cambridge Studies in Low Temperature Physics (Cambridge University Press, Cambridge, England, 2005).

[32] N. P. Proukakis and B. Jackson, J. Phys. B 41, 203002 (2008). 
[33] For videos of the vortex dynamics see https://www.thphys .uni-heidelberg.de/ holography/holoDGPE/.

[34] H. E. Hall and W. F. Vinen, Proc. R. Soc. A 238, 204 (1956).

[35] S. V. Iordanskiǔ, Ann. Phys. (N.Y.) 29, 335 (1964).

[36] S. V. Iordanskiı̌, J. Exp. Theor. Phys. 22, 160 (1966).

[37] See Supplemental Material at http://link.aps.org/ supplemental/10.1103/PhysRevLett.127.101601 for further details, which includes Refs. [38-92].

[38] T. Albash and C. V. Johnson, Phys. Rev. D 80, 126009 (2009).

[39] D. T. Son and A. O. Starinets, J. High Energy Phys. 09 (2002) 042.

[40] P. Breitenlohner and D. Z. Freedman, Phys. Lett. 115B, 197 (1982).

[41] P. Breitenlohner and D. Z. Freedman, Ann. Phys. (N.Y.) 144, 249 (1982).

[42] D. D. Solnyshkov, H. Terças, K. Dini, and G. Malpuech, Phys. Rev. A 89, 033626 (2014).

[43] I. Bloch, J. Dalibard, and W. Zwerger, Rev. Mod. Phys. 80, 885 (2008).

[44] Z. Hadzibabic, P. Krüger, M. Cheneau, S. P. Rath, and J. Dalibard, New J. Phys. 10, 045006 (2008).

[45] T. W. Neely, A. S. Bradley, E. C. Samson, S. J. Rooney, E. M. Wright, K. J. H. Law, R. Carretero-González, P. G. Kevrekidis, M. J. Davis, and B. P. Anderson, Phys. Rev. Lett. 111, 235301 (2013).

[46] W. J. Kwon, G. Moon, J.-y. Choi, S. W. Seo, and Y.-i. Shin, Phys. Rev. A 90, 063627 (2014).

[47] D. S. Petrov and G. V. Shlyapnikov, Phys. Rev. A 64, 012706 (2001).

[48] C. Mora and Y. Castin, Phys. Rev. Lett. 102, 180404 (2009).

[49] R. A. Duine and H. T. C. Stoof, Phys. Rev. A 65, 013603 (2001).

[50] S. P. Cockburn and N. P. Proukakis, in Quantum Gases: Finite Temperature and Non-Equilibrium Dynamics (Imperial College Press, London, 2013), p. 177.

[51] E. Zaremba, T. Nikuni, and A. Griffin, J. Low Temp. Phys. 116, 277 (1999).

[52] A. J. Allen, C. F. Barenghi, N. P. Proukakis, and E. Zaremba, in Quantum Gases: Finite Temperature and Non-Equilibrium Dynamics (Imperial College Press, London, 2013), p. 93.

[53] S. Lan, W. Chen, X. Liang, J. Chen, and X. Liu, arXiv: 2003.01376.

[54] V. Ambegaokar, B. I. Halperin, D. R. Nelson, and E. D. Siggia, Phys. Rev. Lett. 40, 783 (1978).

[55] E. B. Sonin, Rev. Mod. Phys. 59, 87 (1987).

[56] V. Ambegaokar, B. I. Halperin, D. R. Nelson, and E. D. Siggia, Phys. Rev. B 21, 1806 (1980).

[57] E. B. Sonin, Phys. Rev. B 55, 485 (1997).

[58] D. J. Thouless, P. Ao, and Q. Niu, Phys. Rev. Lett. 76, 3758 (1996).

[59] L. Thompson and P. C. E. Stamp, Phys. Rev. Lett. 108, 184501 (2012).

[60] T. Cox and P. C. E. Stamp, J. Low Temp. Phys. 171, 459 (2013).

[61] G. Blatter, M. V. Feigel'man, V. B. Geshkenbein, A. I. Larkin, and V. M. Vinokur, Rev. Mod. Phys. 66, 1125 (1994).
[62] P. Rosenbusch, V. Bretin, and J. Dalibard, Phys. Rev. Lett. 89, 200403 (2002).

[63] J. R. Abo-Shaeer, C. Raman, and W. Ketterle, Phys. Rev. Lett. 88, 070409 (2002).

[64] E. A. L. Henn, J. A. Seman, G. Roati, K. M. F. Magalhães, and V. S. Bagnato, Phys. Rev. Lett. 103, 045301 (2009).

[65] L. Onsager, Nuovo Cimento 6, 279 (1949).

[66] I. S. Aranson and L. Kramer, Rev. Mod. Phys. 74, 99 (2002).

[67] E. Miot, Anal. PDE 2, 159 (2009).

[68] M. Kurzke, C. Melcher, R. Moser, and D. Spirn, Indiana University Mathematics Journal 58, 2597 (2009).

[69] N. Iqbal and H. Liu, Classical Quantum Gravity 29, 194004 (2012).

[70] K. W. Schwarz, Phys. Rev. B 38, 2398 (1988).

[71] A. T. A. M. de Waele and R. G. K. M. Aarts, Phys. Rev. Lett. 72, 482 (1994).

[72] J. Koplik and H. Levine, Phys. Rev. Lett. 71, 1375 (1993).

[73] S. Nazarenko and R. West, J. Low Temp. Phys. 132, 1 (2003).

[74] R. Tebbs, A. J. Youd, and C. F. Barenghi, J. Low Temp. Phys. 162, 314 (2011).

[75] G. P. Bewley, M. S. Paoletti, K. R. Sreenivasan, and D. P. Lathrop, Proc. Natl. Acad. Sci. U.S.A. 105, 13707 (2008).

[76] M. Paoletti, M. E. Fisher, and D. Lathrop, Physica (Amsterdam) 239D, 1367 (2010).

[77] G. Agnolet, D. F. McQueeney, and J. D. Reppy, Phys. Rev. B 39, 8934 (1989).

[78] A. I. Golov and P. M. Walmsley, J. Low Temp. Phys. 156, 51 (2009).

[79] E. Kozik and B. Svistunov, Phys. Rev. Lett. 100, 195302 (2008).

[80] P. O. Fedichev and G. V. Shlyapnikov, Phys. Rev. A 60, R1779 (1999).

[81] M. Kobayashi and M. Tsubota, Phys. Rev. Lett. 97, 145301 (2006).

[82] N. G. Berloff and A. J. Youd, Phys. Rev. Lett. 99, 145301 (2007).

[83] B. Jackson, N. P. Proukakis, C. F. Barenghi, and E. Zaremba, Phys. Rev. A 79, 053615 (2009).

[84] A. M. J. Schakel, Boulevard of Broken SymmetriesEffective Field Theories of Condensed Matter (World Scientific, Singapore, 2008).

[85] C. Pethick and H. Smith, Bose-Einstein Condensation in Dilute Gases (Cambridge University Press, Cambridge, England, 2006).

[86] K. Maeda and T. Okamura, Phys. Rev. D 78, 106006 (2008).

[87] A. Galántai, J. Comput. Appl. Math. 124, 25 (2000).

[88] G. Krstulovic, Phys. Rev. E 86, 055301(R) (2012).

[89] G. Krstulovic, M. Brachet, and E. Tirapegui, Phys. Rev. E 78, 026601 (2008).

[90] A. Villois, G. Krstulovic, D. Proment, and H. Salman, J. Phys. A 49, 415502 (2016).

[91] I. C. Christov, R. J. Decker, A. Demirkaya, V. A. Gani, P. G. Kevrekidis, and R. V. Radomskiy, Phys. Rev. D 99, 016010 (2019).

[92] I. C. Christov, R. J. Decker, A. Demirkaya, V. A. Gani, P. G. Kevrekidis, A. Khare, and A. Saxena, Phys. Rev. Lett. 122, 171601 (2019). 
[93] L. Tisza, Nature (London) 141, 913 (1938).

[94] L. Landau, Phys. Rev. 60, 356 (1941).

[95] S. S. Gubser and A. Nellore, Phys. Rev. D 80, 105007 (2009).

[96] To distinguish quantities denoted by the same letter, they are written with a tilde in holography and without in DGPE.

[97] Z. Hadzibabic and J. Dalibard, in Nano Optics and Atomics: Transport of Light and Matter Waves, Proceedings of the International School of Physics "Enrico Fermi", Course CLXXIII, Varenna, 2009, edited by R. Kaiser and D. Wiersma (IOS Press, Amsterdam, 2009).

[98] D. S. Petrov, M. Holzmann, and G. V. Shlyapnikov, Phys. Rev. Lett. 84, 2551 (2000).

[99] P. Naidon, E. Tiesinga, W. F. Mitchell, and P. S. Julienne, New J. Phys. 9, 19 (2007).

[100] V. L. Berezinskiŭ, J. Exp. Theor. Phys. 34, 610 (1972).
[101] J. Kosterlitz and D. Thouless, J. Phys. C 6, 1181 (1973).

[102] R. A. Duine, B. W. A. Leurs, and H. T. C. Stoof, Phys. Rev. A 69, 053623 (2004).

[103] P. Makotyn, C. E. Klauss, D. L. Goldberger, E. A. Cornell, and D. S. Jin, Nat. Phys. 10, 116 (2014).

[104] D. S. Petrov, C. Salomon, and G. V. Shlyapnikov, Phys. Rev. Lett. 93, 090404 (2004).

[105] G. Moon, W. J. Kwon, H. Lee, and Y.-i. Shin, Phys. Rev. A 92, 051601(R) (2015).

[106] M. Kim and W. I. Glaberson, Phys. Rev. Lett. 52, 53 (1984).

[107] P. W. Adams and W. I. Glaberson, Phys. Rev. B 35, 4633 (1987).

[108] D. Finotello, Y. Y. Yu, and F. M. Gasparini, Phys. Rev. B 41, 10994 (1990).

[109] T. Oda, M. Hieda, R. Toda, T. Matsushita, and N. Wada, J. Low Temp. Phys. 158, 262 (2010). 